\title{
Research Article \\ Study on Dynamic Stall Control of Rotor Airfoil Based on Coflow Jet
}

\author{
Jiaqi Liu $\mathbb{D}^{1},{ }^{1}$ Rongqian Chen $\mathbb{D}^{1},{ }^{1}$ Ruofan Qiu, ${ }^{1}$ Yancheng You, ${ }^{1}$ and Weiguo Zhang ${ }^{2}$ \\ ${ }^{1}$ School of Aerospace Engineering, Xiamen University, Xiamen 361005, China \\ ${ }^{2}$ Rotor Aerodynamics Key Laboratory, China Aerodynamics Research and Development Center, Mianyang 621000, China \\ Correspondence should be addressed to Rongqian Chen; rqchen@xmu.edu.cn
}

Received 7 August 2020; Revised 10 November 2020; Accepted 2 December 2020; Published 16 December 2020

Academic Editor: Erkan Kayacan

Copyright (C) 2020 Jiaqi Liu et al. This is an open access article distributed under the Creative Commons Attribution License, which permits unrestricted use, distribution, and reproduction in any medium, provided the original work is properly cited.

\begin{abstract}
In this study, a dynamic stall control strategy, called the co-flow jet (CFJ), is applied to the rotor airfoil. The effect of the CFJ on the unsteady dynamic stall characteristics of the rotor airfoil is numerically investigated via numerical simulations of the unsteady Reynolds-averaged Navier-Stokes (URANS) equations coupled with the Spalart-Allmaras (S-A) turbulence model. The numerical methods are validated by a NACA0012 pitching airfoil case and a NACA6415 airfoil case based on the CFJ, and good agreement with experiments is found. Via the study of the typical conditions of CFJ control to suppress the dynamic stall of the OA212 rotor airfoil, it is verified that this method has a good effect on dynamic stall suppression. The diffusion and blending of the turbulent shear layer between the CFJ injection jet and the main flow excite the main flow and enhance its ability to resist the reverse pressure gradient; this suppresses the generation and development of the separation vortex, thereby enhancing the aerodynamic characteristics, improving the hysteresis effect, and increasing the system stability. On this basis, the control parameters of the CFJ are further studied, including the influences of the jet momentum coefficient and the positions and sizes of the injection and suction slots on suppressing the dynamic stall of the rotor airfoil. It is found that there is a jet momentum coefficient that optimizes the suppression effect of the dynamic stall of the rotor airfoil. Moreover, the position of the injection slot is found to have a greater effect on the dynamic stall suppression, while the size of the injection slot and the position and size of the suction slot have little effect.
\end{abstract}

\section{Introduction}

As compared with a fixed-wing aircraft, helicopters have unique advantages, such as vertical take-off and landing, high maneuverability, and hovering in the air, and they are widely used in both military and civilian fields. However, the low flight speed of conventional helicopters greatly limits their application range. The limitation of the forward flight speed of helicopters is closely related to the flow characteristics of the airflow on the rotor blade surface [1-3]. The retreating blades of the rotor generally work in an aerodynamic environment with a large angle of attack (AoA), and the complex unsteady dynamic stall phenomenon caused by the AoA of most sections of the blade exceeds its stall AoA. The dynamic stall of three-dimensional rotors $[4,5]$ and two-dimensional airfoils [6-8] is investigated which indicates that the dynamic stall has a great influence on their aerodynamic performance.
The dynamic stall leads to sharp increases in the drag coefficient and moment coefficient of the rotor blades, which seriously restricts the improvement of the aerodynamic performance and forward flight speed of helicopters. Moreover, the obvious aerodynamic hysteresis effect of the rotor blade caused by dynamic stall will lead to stall flutter, a surge in vibration load, noise enhancement, etc. [9], which will introduce many adverse effects to helicopters. Therefore, it is of great significance to research the methods and mechanisms of rotor dynamic stall suppression.

The current flow control methods of the suppression of the dynamic stall of the rotor include both passive and active control methods. Passive control methods include vortex generators [10-12], Gurney flaps [13-15], fixed leading-edge slats $[16,17]$, and fixed-droop leading edges [18]. However, these methods only have a good control effect on the suppression of the dynamic stall of the 
rotor under the design conditions; when the actual conditions deviate from the design conditions, the control effect is poor. Active control methods have many advantages over passive control methods; they can be used at the required time and position and can be actively adjusted. Active control methods primarily include active trailingedge flaps [19-22], dynamic-droop leading edges [23-25], synthetic jets [26-28], and plasma jets [29-31]. However, active trailing-edge flaps and dynamic-droop leading edges tend to cause obvious changes to the center of gravity and load [32]. Moreover, they always require a complex mechanical adjustment mechanism and control systems. The synthetic jets and plasma jets interact weakly with the mainstream, so it is difficult to achieve continuous and efficient control. In summary, the dynamic stall problem of the rotor has not been sufficiently solved, and there is an urgent need to develop a more flexible and effective flow control method.

The co-flow jet (CFJ) flow control technique was developed in recent years by Zha et al. [33-36]. The CFJ airfoil is designed with an injection slot near the leading edge and a suction slot near the trailing edge of the airfoil. The injection slot injects a high-energy tangential jet in the same direction as the main flow, and the suction slot sucks in the same mass flow. Pumps and pipes are arranged inside the airfoil to transport the air sucked in from the suction slot to the injection slot. The diffusion and blending of the turbulent shear layer with the main flow excite the main flow and enhance its ability to resist the reverse pressure gradient. The results of a wind tunnel test of static airfoil [34] and numerical simulation [37] have demonstrated that CFJ can suppress flow separation, improve stall characteristics, and significantly increase the lift-to-drag ratio of the airfoil and are also characterized by a high energy utilization rate. Unlike the traditional single-hole synthetic jet, the CFJ can realize a continuous jet and flexibly control the jet intensity. Moreover, because the blowing volume and the suction volume are equal, this method is also a flow control method without an additional air source.

Based on the preceding analysis, the CFJ is a promising method by which to suppress the dynamic stall of retreating rotor blades. However, there have been few studies on the application of this method to rotor dynamic stall. Only a few studies preliminarily verified that CFJ could improve the aerodynamic performance and suppress the dynamic stall [38-41]. But its control effect and mechanism, influencing factors, etc., must all be further investigated. Therefore, in this work, the CFJ is applied to the suppression of the dynamic stall of the trailing blades of the rotor. The OA212 rotor airfoil is taken as the research object, and the method of solving the Navier-Stokes equation based on the sliding mesh technique is employed to numerically simulate the dynamic stall. The CFJ control is applied to the airfoil, and it is verified that this method can suppress the dynamic stall. The effects of the control parameters of the CFJ on suppressing the dynamic stall of the rotor airfoil are then investigated and include the jet momentum coefficient and the position and size of the injection and suction slots; the findings lay a founda- tion for the research on the dynamic stall control of actual rotor blades.

\section{Numerical Methods}

2.1. Governing Equations. The Ansys Fluent is utilized in this work to simulate the dynamic stall of the rotor airfoil, and the two-dimensional compressible Reynolds-averaged NavierStokes equations are employed, which can be written as

$$
\frac{\partial}{\partial t} \int_{\Omega} \mathbf{W} d \Omega+\oint_{\partial \Omega}\left(\mathbf{F}_{\mathrm{c}}-\mathbf{F}_{\mathrm{v}}\right) d S=0
$$

where $\mathbf{W}$ represents conserved variables and $\mathbf{F}_{c}$ and $\mathbf{F}_{\mathrm{v}}$ are convective fluxes and viscous fluxes, respectively. They are expressed as

$$
\begin{gathered}
\mathbf{W}=\left[\begin{array}{c}
\rho \\
\rho u \\
\rho v \\
\rho E
\end{array}\right], \\
\mathbf{F}_{c}=\left[\begin{array}{c}
\rho \mathbf{V}_{r} \\
\rho u \mathbf{V}_{r}+n_{x} p \\
\rho v \mathbf{V}_{r}+n_{y} p \\
\rho H \mathbf{V}_{r}+\mathbf{V}_{t} p
\end{array}\right], \\
0 \\
\mathbf{F}_{\mathbf{v}}=\left[\begin{array}{c}
n_{x} \tau_{x x}+n_{y} \tau_{x y} \\
n_{x} \tau_{y x}+n_{y} \tau_{y y} \\
n_{x} \Theta_{x}+n_{y} \Theta_{y}
\end{array}\right],
\end{gathered}
$$

where $u$ and $v$ represent the Cartesian velocity components; $\rho$ and $p$ represent the density and pressure of airflow, respectively; $E$ and $H$ represent the total energy and total enthalpy per unit mass, respectively; $V_{r}=V-V_{t} ; V$ is the absolute velocity; $V_{t}$ is the contravariant velocity; $\tau_{i j}$ denotes the viscous stress; and $\Theta_{i}$ is the term describing the heat conduction in the fluid [42].

The governing equations are solved by the finite volume method. The Roe scheme is used to discretize the inviscid fluxes, and the second-order central difference is used to compute the viscous fluxes. To simulate the flow field of the rotor airfoil under dynamic stall conditions, the dual timestepping approach is employed to solve the temporal discretization [43]. The Spalart-Allmaras (S-A) turbulence model is used to calculate the turbulence viscosity [44]. The noslip boundary condition is enforced on the rotor airfoil surface, and the pressure far-field boundary condition is set on the far-field boundary.

2.2. Sliding Mesh Technique. The sliding mesh technique is adopted for the numerical simulation of the pitching motion of the rotor airfoil, and the computational domain, which includes both the fixed and rotating domains, is 


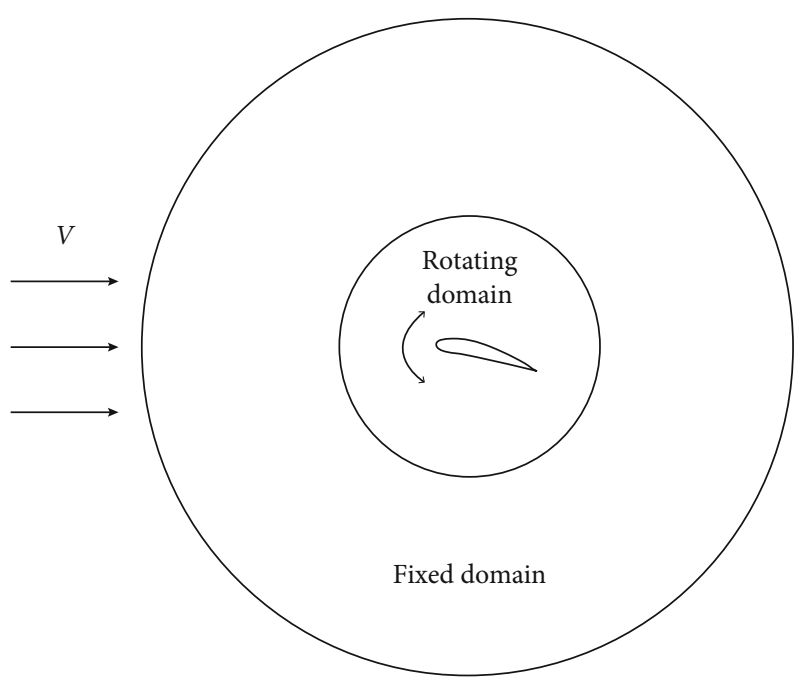

FIGURE 1: The schematic diagram of the computational domain of the sliding mesh technique.

presented in Figure 1. The shape of the rotating domain is a circle with a diameter of 10 times the chord length, and the airfoil is placed in the middle. The shape of the fixed domain is a ring with an inner diameter of 10 times the chord length and an outer diameter of 100 times the chord length. The pitching motion of the airfoil can only be realized by rotating the inner domain during the process of numerical simulation. The flow-field information is transferred between the static domain and the rotating domain via data interpolation on both sides of the interface. This method does not need to deform, regenerate, or dig holes during the process of moving (slipping) between different sets of meshes; it is a simple and efficient method by which to deal with motion boundary problems such as rotation and translation.

2.3. CFJ Simulation Method. The CFJ airfoil is presented in Figure 2. The injection slot is placed near the leading edge of the airfoil, and the suction slot is placed near the trailing edge of the airfoil. There is a micropump inside the airfoil, and the injection slot and the suction slot are connected by pipes. When the pump is turned on, the airflow is ejected from the injection slot in the tangential direction of the wall to form a momentum jet, which is injected into the main flow. Simultaneously, the airflow with the same mass flow will also be sucked into the suction slot. A simplified CFJ calculation model in which the internal pipes and pumps are removed is used in this work. The effect of the CFJ is simulated by applying boundary conditions. The velocity inlet boundary condition is adopted for the injection slot, and the initial speed is calculated by the momentum coefficient and the size of the slot. Then, the speed of the injection slot is adjusted to reach the prescribed $C \mu$, which is an iterative process. The pressure outlet boundary condition is adopted for the suction slot, and the mass flow of the injection slot and the suction slot are matched by adjusting the static pressure of the suction slot.

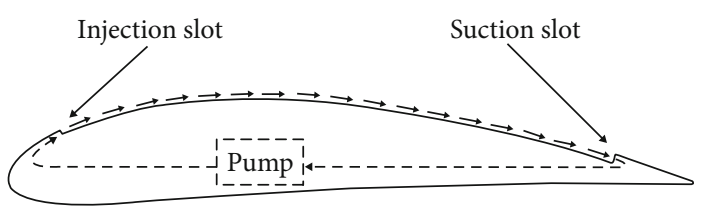

Figure 2: Coflow jet airfoil.

For the CFJ method, the jet momentum coefficient is usually used to reflect the jet intensity, which is defined as

$$
C \mu=\frac{\dot{m} V_{j}}{1 / 2 \rho_{\infty} V_{\infty}^{2} \cdot S},
$$

where $\dot{m}$ is the mass flow rate, $V_{j}$ is the velocity of airflow at the injection slot, $\rho_{\infty}$ is the freestream density, $V_{\infty}$ is the freestream velocity, and $S$ is the reference area of the airfoil.

In this paper, the real variable pitch law of the retreating blade of the rotor is adopted as the motion law of the rotor airfoil based on CFJ control. The function of the AoA $\alpha$ is written as

$$
\alpha(t)=\alpha_{0}+\alpha_{m} \cdot \sin (w t)
$$

where $\alpha_{0}$ is the mean of the AoA, $\alpha_{m}$ is the amplitude of oscillation, and $\omega$ is the angular velocity of oscillation. The center of rotation is at the chord line 0.25 times the chord length from the leading edge. The dimensionless parameter reduced frequency $k$ is generally used to describe the degree of the unsteady influence of oscillating motion and is defined as $k$ $=(\omega c) /\left(2 V_{\infty}\right)$, where $c$ is the chord length.

2.4. Simulation Method Validation. To verify the correctness of the numerical simulation method of rotor dynamic stall based on CFJ control used in this paper, a pitching NACA0012 airfoil case and the suppression of the flow separation of a NACA6415 airfoil based on the steady CFJ are simulated. The numerical simulation results are compared with references.

2.4.1. Dynamic Stall of a NACA0012 Airfoil. A dynamic stall case of a NACA0012 airfoil was simulated to verify the effectiveness of the CFD method for dynamic stall suppression. The flow conditions [45] were a Mach number $\mathrm{Ma}=0.283$ and the chord-based Reynolds number $\operatorname{Re}=3.45 \times 10^{6}$. The law of pitch motion was that given by Eq. (4); additionally, the mean of the AoA $\alpha_{0}=15^{\circ}$, the pitch amplitude $\alpha_{m}=10^{\circ}$, and the reduced frequency $k=0.151$. The numbers of grids in the fixed and rotating domains were 10,881 and 58,738, respectively. The distance from the first grid point to the airfoil surface was $4.95 \times 10^{-6} c$, and $y^{+}$was less than 1.0 . Figure 3 presents the lift coefficient $\mathrm{Cl}$ and the drag coefficient $\mathrm{Cd}$ of the airfoil, from which it can be seen that the calculated hysteresis loops of the lift coefficient and drag coefficient with the AoA are in good agreement with the experimental results; this verifies the correctness of the numerical simulation method for the dynamic stall of the rotor airfoil. 


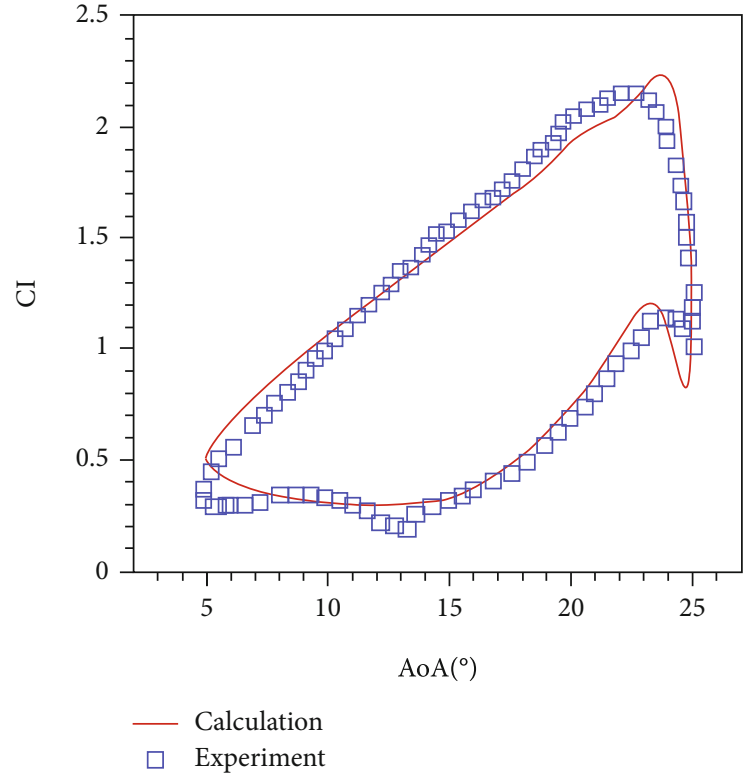

(a) Lift coefficient

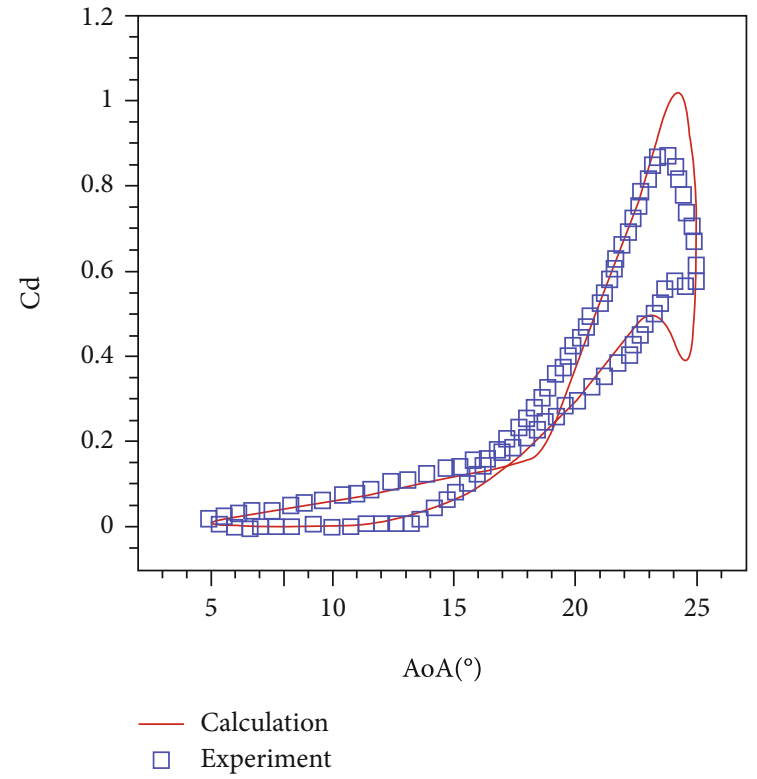

(b) Drag coefficient

Figure 3: Lift and drag coefficient distributions of a NACA0012 airfoil.

2.4.2. Steady CFJ Control for a NACA6415 Airfoil. The NACA6415-CFJ airfoil from a University of Miami wind tunnel experiment was selected as the validation case [46]. The computation conditions were a Mach number of the freestream $\mathrm{Ma}=0.3$, the chord-based Reynolds number Re $=2.08 \times 10^{6}$, and the jet momentum coefficient $C_{\mu}=0.08$. The mesh size of cells was 57,165 , and the height of the first grid point near the airfoil surface was about $6 \times 10^{-6} c ; y^{+}$ was less than 1.0.

The airfoil lift coefficient $\mathrm{Cl}$ and drag coefficient $\mathrm{Cd}$ with the respective AoAs of $0^{\circ}, 5^{\circ}, 10^{\circ}, 15^{\circ}, 20^{\circ}$, and $25^{\circ}$ are presented in Figure 4. The calculation results both with and without the CFJ control were found to be in good agreement with the results of a previous study [47], which verifies the effectiveness of the calculation method for the CFJ used in this paper. From the Mach number contours and the streamlines around the airfoil presented in Figure 5, it is evident that the flow adhesion on the suction surface of the airfoil under CFJ control was greatly enhanced. It can also be found that flow separation occurred at the trailing edge of the airfoil, and the flow separation region of the CFJ airfoil was notably less than that of the baseline airfoil.

\section{Dynamic Stall Control via CFJ under Typical Conditions}

3.1. Computational Model and Grid. The OA212 rotor airfoil was used to study dynamic stall control by the CFJ, and the influences of the control parameters on the dynamic stall and the mechanism of control were analyzed. On this basis, the influences of the CFJ control parameters on dynamic stall control were further analyzed. The control parameters include the jet moment coefficient, injection slot position $x_{1}$
, injection slot size $h_{1}$, suction slot position $x_{2}$, and suction slot size $h_{2}$, as presented in Figure 6 . The flow conditions are listed as follows [48]: the Mach number of freestream $\mathrm{Ma}=0.14$ and the chord-based Reynolds number $\mathrm{Re}=2.7$ $\times 10^{6}$. The law of pitch motion was that given by Eq. (4). Additionally, the mean of the AoA $\alpha_{0}=15^{\circ}$, the pitch amplitude $\alpha_{m}=10^{\circ}$, and the reduced frequency $k=0.105$.

The control parameters of the CFJ under the typical conditions were as follows. The injection slot was placed at $8 \% c$ from the leading-edge point, the size of the injection slot was $0.5 \% c$, the suction slot was placed at $70 \% c$ from the leading-edge point, the size of the suction slot was $1 \% c$, and the CFJ jet momentum coefficient $C \mu=0.08$. A set of structured grids was applied for calculation. Figure 7 (a) presents the structured grid of the OA212 CFJ airfoil, and Figure 7(b) displays the zoomed-in view of the grid near the airfoil. The numbers of grids of the fixed domain and the rotating domain were 32,636 and 110,686 , respectively. The thickness of the first cell near the airfoil surface was 4.5 $\times 10^{-6} c$, and $y^{+}$was less than 1.0 to accurately capture the surface boundary layer.

3.2. Dynamic Stall Characteristic Analysis. Numerical simulations were carried out on the dynamic stall flow field of the OA212 rotor airfoil (baseline airfoil) and that of the OA212 rotor airfoil based on CFJ control (CFJ airfoil). Figure 8 presents the comparison between the dynamic stall flow fields of the OA212 baseline airfoil and the CFJ airfoil at different times during an oscillation period when the airfoil AoAs were $5^{\circ}, 15^{\circ} \uparrow, 25^{\circ}, 20^{\circ} \downarrow$, and $15^{\circ} \downarrow$, respectively ( $\uparrow$ indicates the airfoil was in the upward phase; $\downarrow$ indicates the airfoil was in the downward phase). It is evident from the figure that the dynamic stall of the airfoil was mainly dominated by the separation vortex at the leading edge of the airfoil. With the 


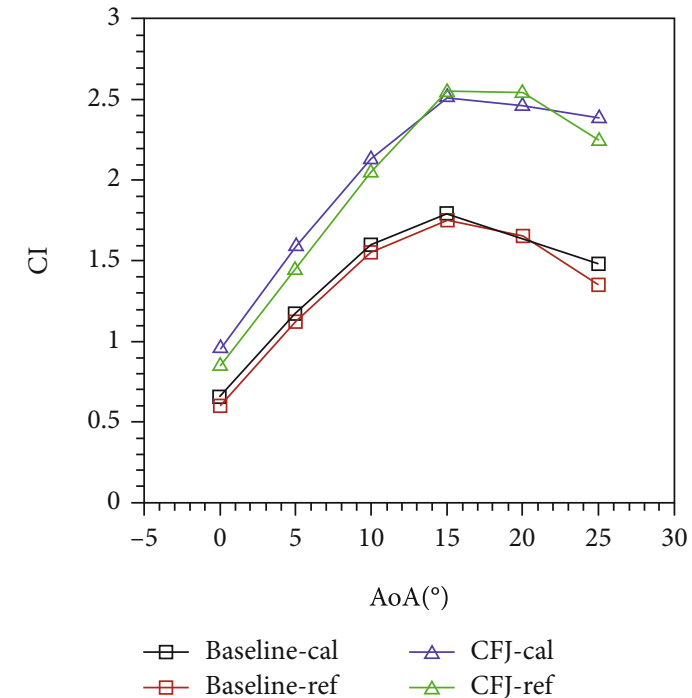

(a) Lift coefficient

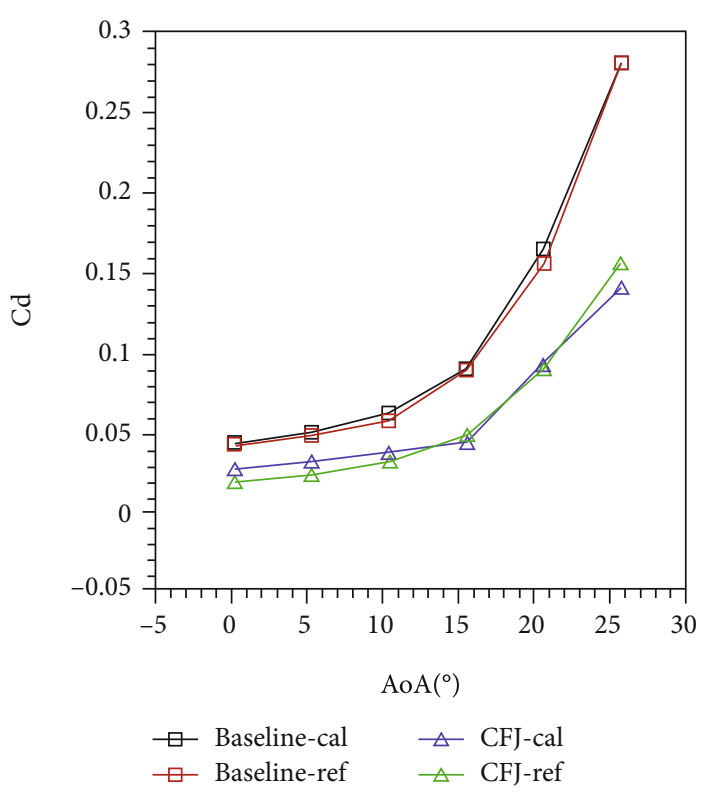

(b) Drag coefficient

Figure 4: Variation of the lift and drag coefficients of the NACA6415 baseline and CFJ airfoils.

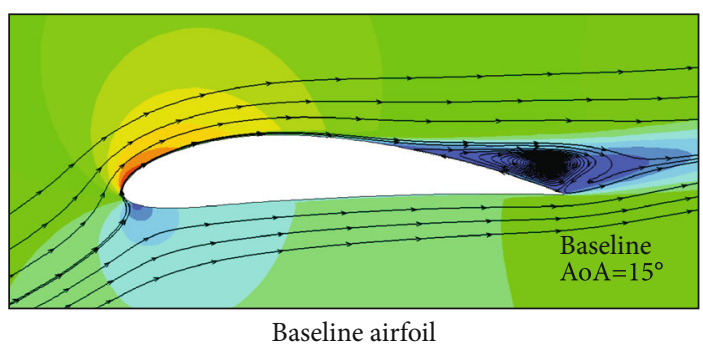

(a)

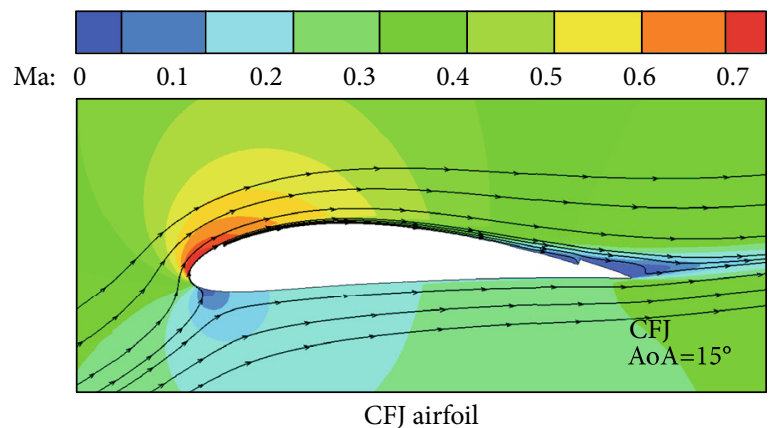

(b)

FIgURE 5: Mach number contours and streamlines of the NACA6415 baseline and CFJ airfoils $\left(\right.$ AoA $\left.=15^{\circ}\right)$.

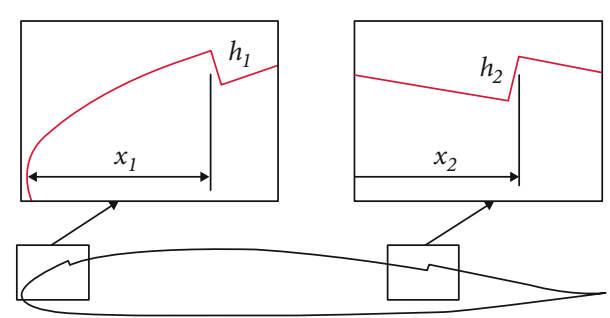

FIGURE 6: Schematic illustration of the OA212 CFJ airfoil geometry.

change of the AoA of the airfoil, the separation vortex at the leading edge moved to the trailing edge of the airfoil and fell off. The CFJ control could well suppress the generation and development of the separation vortex at the leading edge of the airfoil. Moreover, the energy of the jet at the injection slot was mixed with the main flow and was unidirectionally transferred from the jet to the main flow, thereby enhancing the ability of the airflow to overcome the reverse pressure gradient. This greatly enhanced the adhesion of the airflow on the suction surface, which suppressed the dynamic stall of the OA212 airfoil. The airflow velocity of the airfoil suction surface was significantly increased after applying the CFJ control. So, the pressure of the airfoil suction surface decreased, which was beneficial to the increase of the lift of the airfoil. In addition, it can be seen from the flow lines around the airfoil that the stagnation point of the CFJ airfoil under the same condition was significantly shifted back as compared to that of the baseline airfoil, indicating an increased effective AoA. This was also the reason for the significant increase in the lift coefficient of the airfoil.

The hysteresis curves of the lift coefficient and moment coefficient with the AoA within one cycle are presented in Figure 9. It can be seen that the positive peak value of the lift coefficient of the airfoil under CFJ control increased and the negative peak value of the moment coefficient decreased. The lift coefficient increased overall, and the moment coefficient decreased overall, especially during the downward phase of 


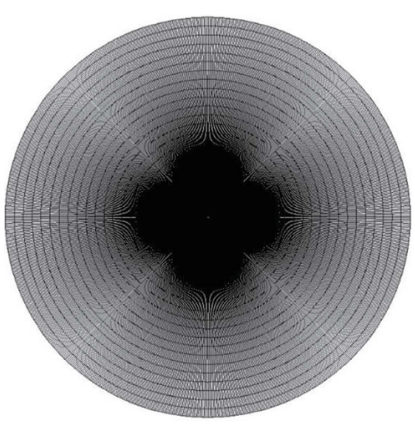

(a) Overall

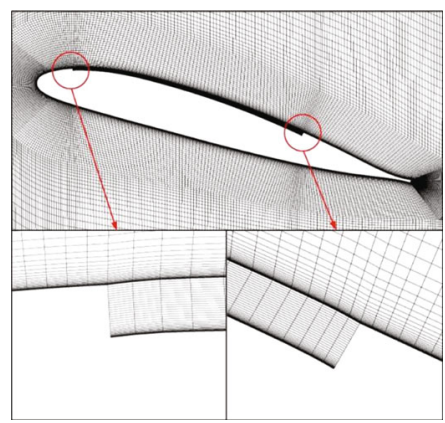

(b) Near the airfoil

Figure 7: Computational grids of the OA212 CFJ airfoil.

the airfoil. The linear sections of the hysteresis curves of the lift coefficient and moment coefficient of the airfoil based on CFJ control were wider, the area of the hysteresis curve was greatly reduced, and the hysteresis effect was obviously weakened. The moment hysteresis curve reflects the aerodynamic damping characteristics of the airfoil and is related to the stability of the system. The curve direction determines whether the system is positively or negatively damped. The area enclosed by the curve in the counterclockwise direction indicates positive damping, while that in the clockwise direction indicates negative damping. During the entire pitching period, if the negative aerodynamic damping exceeds the positive aerodynamic damping, the system will lose stability and stall flutter and other phenomena will occur; otherwise, the system will be stable [49]. From Figure 9(b), it can be observed that the moment coefficient hysteresis curve of the baseline airfoil was crossed during the entire pitching period, and a part of the area representing negative damping appeared, which will adversely affect the stability of the system. In contrast, the aerodynamic damping of the CFJ airfoil was positive during the entire process. Therefore, the method of CFJ control not only increased the overall lift coefficient of the rotor airfoil but also reduced the negative peak of the airfoil pitching moment and the negative aerodynamic damping, thereby effectively increasing the stability of the system.

To further analyze the aerodynamic characteristics of the rotor airfoil in the pitching period, Figure 10 presents the surface pressure coefficient distributions of the OA212 baseline airfoil and CFJ airfoil at different AoAs, where Cp represents the pressure coefficient and $x / c$ is the dimensionless position of the chord. It can be observed from the figure that the area enclosed by the pressure coefficient curve of the CFJ airfoil is larger than that of the baseline airfoil during the entire pitching period, especially during the pitchdownward phase or at high AoAs. This reflects that the lift coefficient of the airfoil after CFJ control will be significantly increased.

\section{The Effects of Different CFJ Parameters on the Dynamic Stall of the Rotor Airfoil}

Based on the previous verification that the CFJ can suppress the dynamic stall of the airfoil, the influences of the CFJ control parameters on the dynamic stall suppression of the
OA212 rotor airfoil are further systematically investigated in this section. The control parameters include the jet momentum coefficient of the CFJ, the position and size of the injection slot, and the position and size of the suction slot.

4.1. Effect of Jet Momentum Coefficient. The influence laws of different jet momentum coefficients $\left(C_{\mu}=0.06, C_{\mu}=0.08, C_{\mu}=0.10\right.$, and $\left.C_{\mu}=0.12\right) \quad$ on the dynamic stall characteristics of the airfoil were studied while keeping the pitch motion law of the airfoil and the other parameters of the CFJ airfoil unchanged. Additionally, the injection slot was located at $8 \% c$, the injection slot size was $0.5 \% c$, the suction slot was located at $70 \% c$, and the suction slot size was $1 \% c$.

Figure 11 presents the Mach number contours and streamlines of the airfoil under CFJ control with different jet flow coefficients $\left(\mathrm{AOA}=25^{\circ}\right)$, from which it is evident that the CFJ with different jet momentum coefficients can well suppress the dynamic stall of the rotor airfoil, and the differences among the flow fields for different control conditions were small. With the increase of the jet momentum coefficient, the airflow velocity between the injection slot at the leading edge and suction slot at the trailing edge increased, and the effect of suppressing separation at the trailing edge of the airfoil was also improved, which is beneficial for the increase of the lift coefficient of the airfoil.

Figure 12 presents the hysteresis curves of the airfoil lift coefficient and moment coefficient with the AoA under CFJ control with different jet momentum coefficients. The lift coefficient of the airfoil increased significantly under the action of different jet momentum coefficients, and the negative peak value of the moment coefficient decreased. However, the change law was not positively correlated with the increase of the jet momentum coefficient; instead, the lift coefficient increased the most when the momentum coefficient $C_{\mu}=0.08$. The linearities of the hysteresis curves of the aerodynamic parameters of the airfoil are similar, the areas enclosed by the curve are relatively close, and the aerodynamic damping is positive, thereby ensuring the stability of the system.

Table 1 reports the degrees of change of the positive peak values of the lift coefficient and the negative peak values of the moment coefficient of the airfoil under CFJ 


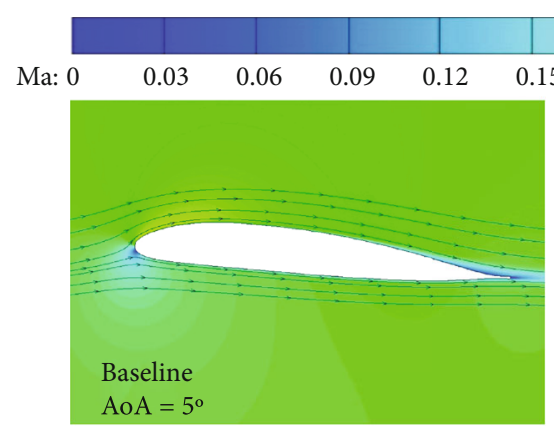

(a) Baseline, AoA $=5^{\circ}$

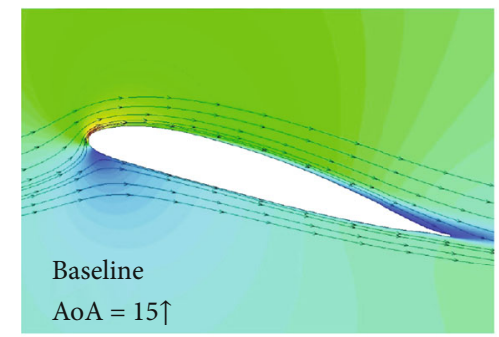

(c) Baseline, AoA $=15^{\circ} \uparrow$

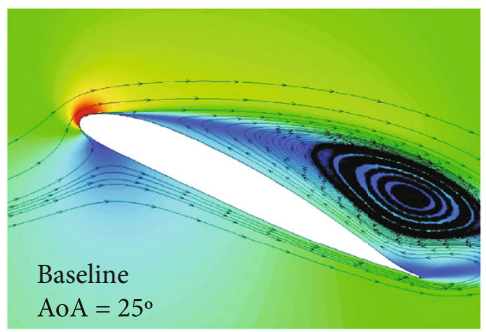

(e) Baseline, AoA $=25^{\circ}$

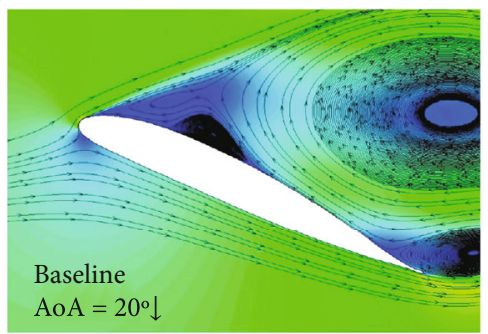

(g) Baseline, AoA $=20^{\circ} \uparrow$

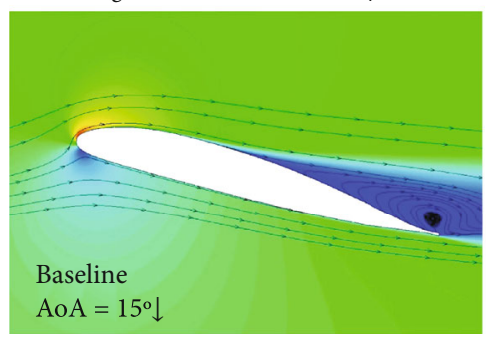

(i) Baseline, AoA $=15^{\circ} \downarrow$
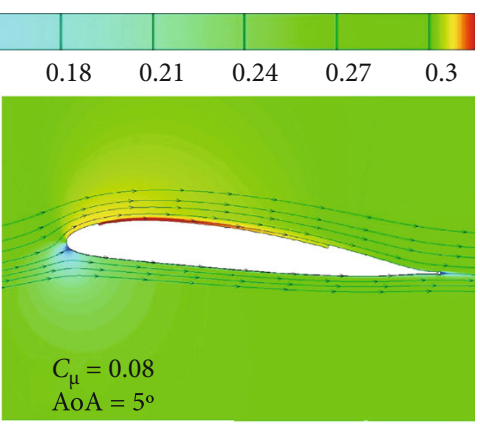

(b) $\mathrm{CFJ}, \mathrm{AoA}=5^{\circ}$

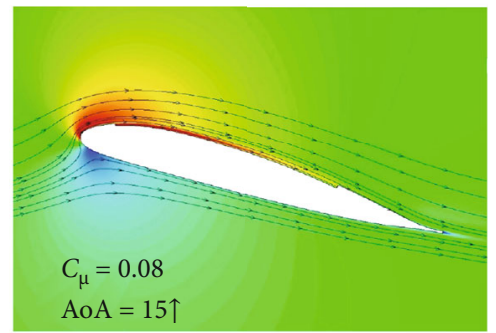

(d) CFJ, AoA $=15^{\circ} \uparrow$

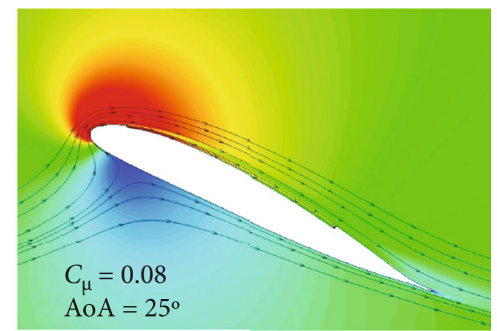

(f) $\mathrm{CFJ}, \mathrm{AoA}=25^{\circ}$

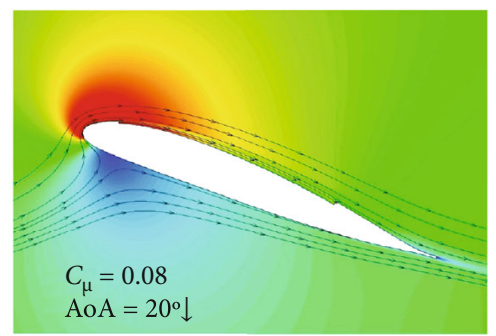

(h) CFJ, AoA $=20^{\circ} \uparrow$

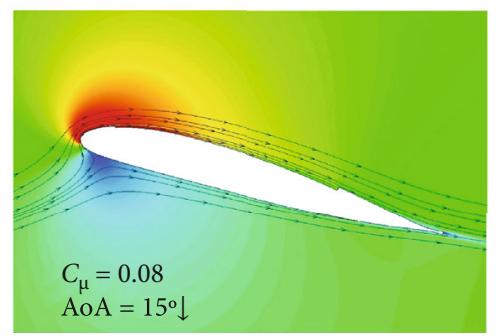

(j) CFJ, $\mathrm{AoA}=15^{\circ} \downarrow$

FIGURE 8: Comparison between the flow fields of the OA212 baseline and CFJ airfoils at different AoAs. 


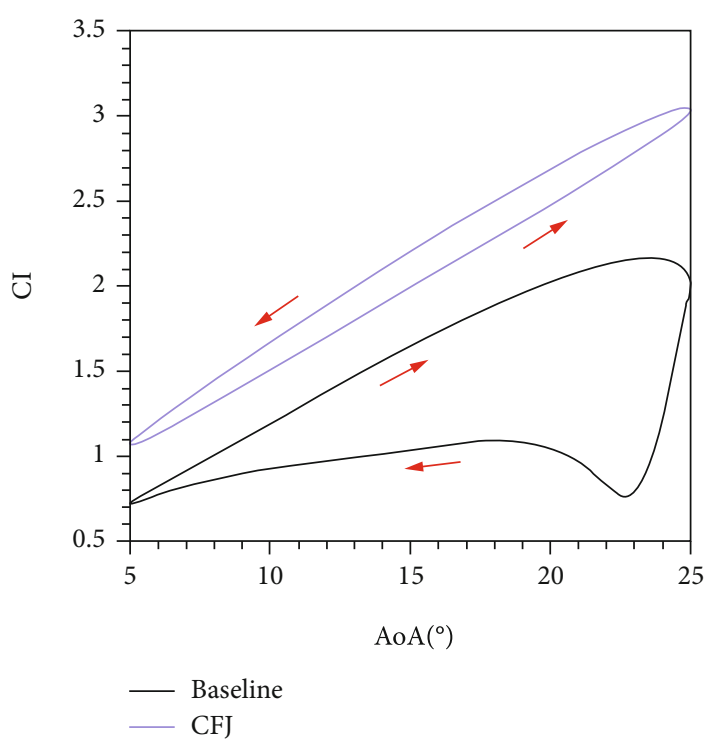

(a) Lift coefficient

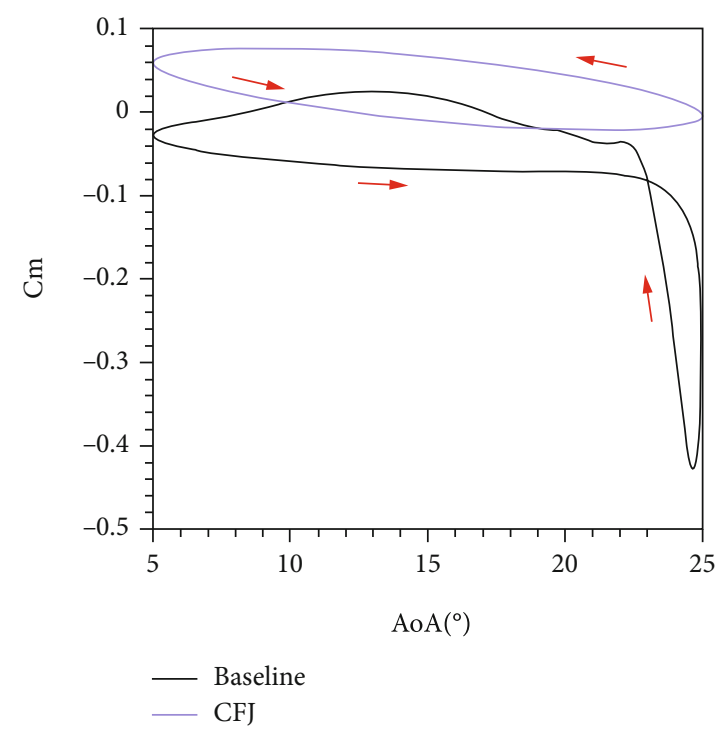

(b) Moment coefficient

FIgURE 9: Lift and moment coefficient distributions of the OA212 baseline and CFJ airfoils.

control with different jet momentum coefficients during a pitching period. The lift coefficient of the airfoil was improved to different degrees under CFJ control, and the negative peak value of the moment coefficient was reduced to different degrees. The average increase in the maximum lift coefficient was about $25 \%$, and the maximum increase was $37.77 \%$ when $C_{\mu}=0.08$. Additionally, the maximum moment coefficient was decreased by about $90 \%$, and the maximum decrease was $94.95 \%$ when $C_{\mu}=0.08$. Therefore, a greater momentum coefficient does not indicate a better control effect on the dynamic stall; instead, the optimal jet flow coefficient will optimize the control effect. Because the CFJ is an active flow control scheme and requires external energy input, a smaller jet momentum coefficient should be selected under the premise of ensuring the control effect to reduce energy consumption.

4.2. Effects of Injection Slot Parameters. The influence of the injection slot position on the dynamic stall of the airfoil was investigated by varying the position of the injection slot from $6 \% c$ to $10 \% c$. The pitch motion law of the airfoil and the other parameters of the CFJ were kept unchanged, and the jet momentum coefficient $C_{\mu}=0.08$.

By comparing the hysteresis curves of the airfoil lift coefficient and moment coefficient with the AoA at different injection slot positions presented in Figure 13, it is evident that the injection slot position had a greater influence on the control effect of the dynamic stall of the airfoil. The best effect was achieved when the injection slot was placed at 9\% $c$; the peak value of the maximum lift coefficient was increased by $103.13 \%$, and the peak moment coefficient was decreased by $86.36 \%$. The curves of the lift coefficient and moment coefficient with the AoA under other conditions are relatively similar.
By analyzing the changes in the dynamic flow field of the OA212 baseline airfoil, it can be determined that when the airfoil moved to the maximum AoA of $25^{\circ}$, the upper surface airflow separation was exactly at $9 \% c$ of the leading edge, as presented in Figure 14. Therefore, when the CFJ injection slot is arranged near the separation point of the airfoil leading edge, the flow separation can be well suppressed, and the control effect will be optimal when the injection slot is at the separation point.

The influence of the injection slot size on the dynamic stall of the airfoil was studied by varying the size of the injection slot from $0.3 \% c$ to $0.7 \% c$. The pitch motion law of the airfoil and the other parameters of the CFJ airfoil were kept unchanged, and the jet flow coefficient $C_{\mu}=0.08$.

Figure 15 presents the hysteresis curves of the lift coefficient and moment coefficient of the CFJ airfoil with the AoA under different injection slot sizes. The size of the injection slot had little impact on the control effect of the dynamic stall of the airfoil. However, when the airfoil approached the maximum AoA of $25^{\circ}$, with the increase of the size of the air outlet, the maximum value of the lift coefficient decreased slightly, and the change of the moment coefficient was small. This demonstrates that the size of the injection slot only has a slight influence on the control effect of the high-AoA condition.

4.3. Effect of Suction Slot Parameters. The influence of the suction slot position on the dynamic stall characteristics of the airfoil was investigated by varying the position of the suction slot from $60 \% c$ to $80 \% c$. The pitch motion law of the airfoil and the other parameters of the CFJ airfoil were kept unchanged, and the jet momentum coefficient $C_{\mu}=0.08$.

Figure 16 presents the hysteresis curves of the airfoil lift coefficient and moment coefficient with the AoA at different suction slot positions. The correlation between the position 


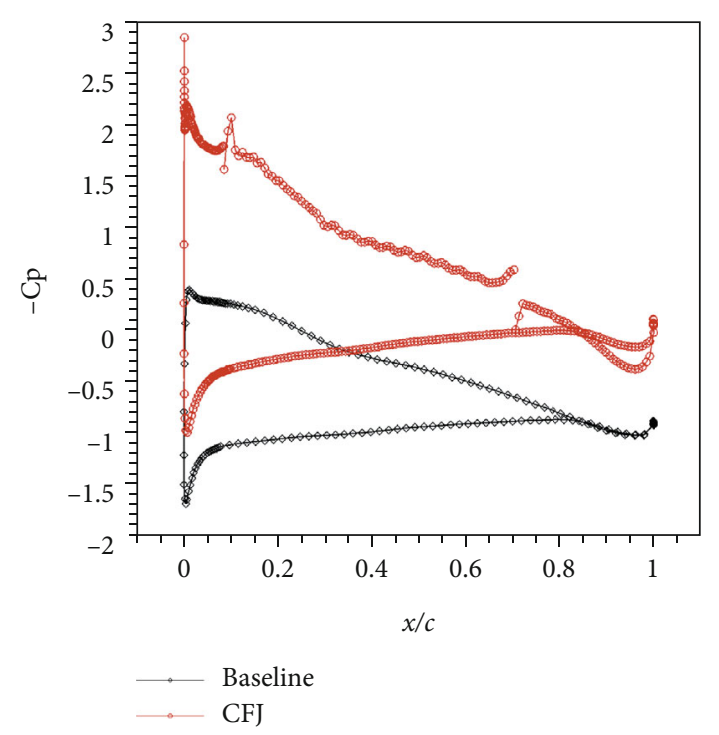

(a) $\mathrm{AoA}=5^{\circ}$

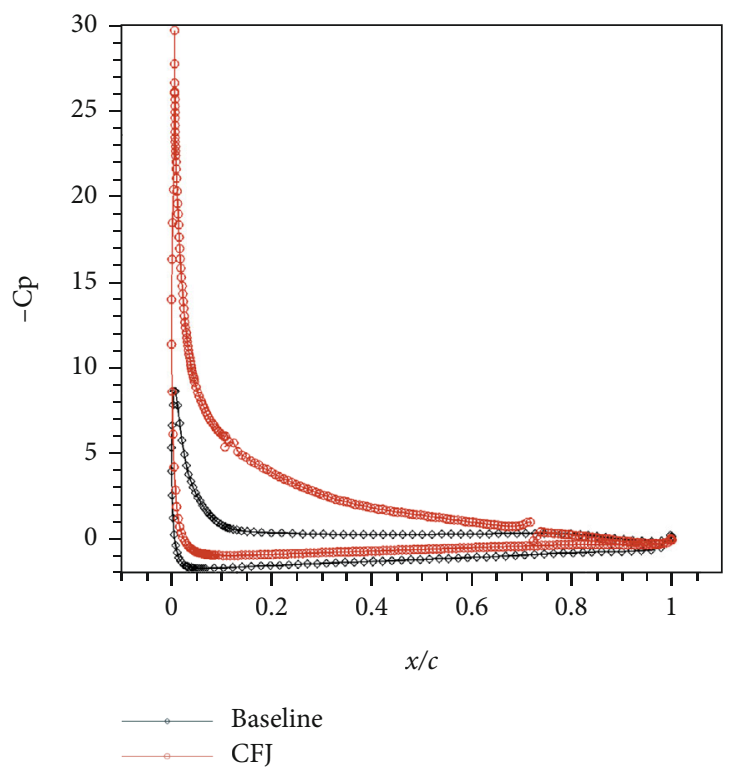

(c) $\mathrm{AoA}=25^{\circ}$

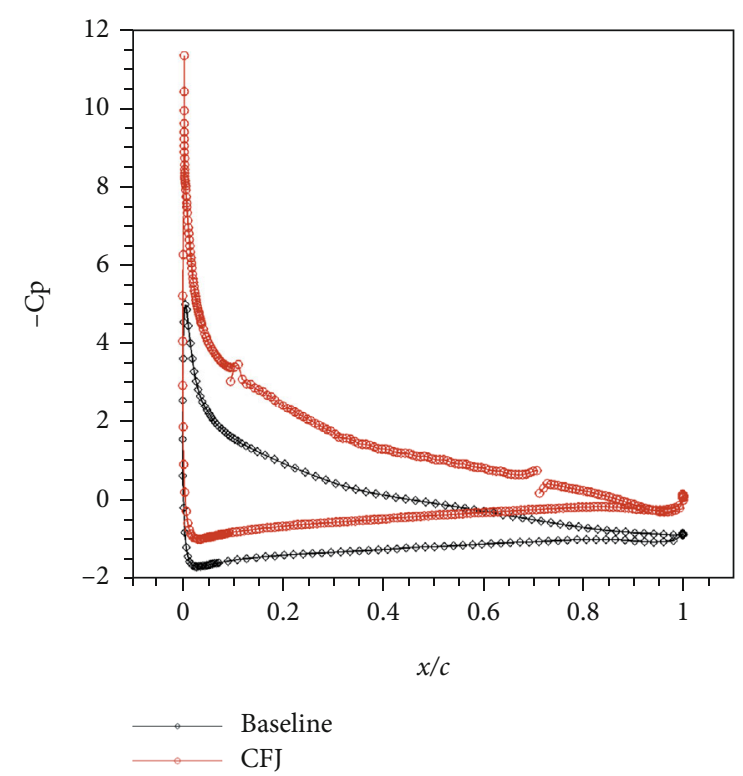

(b) $\mathrm{AoA}=15^{\circ} \uparrow$

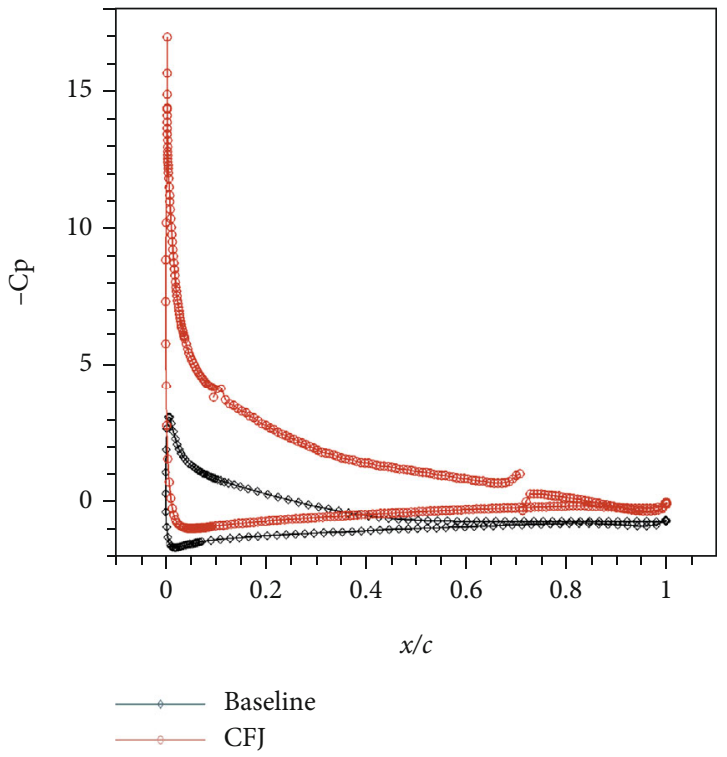

(d) $\mathrm{AoA}=15^{\circ} \downarrow$

Figure 10: Comparison between the pressure coefficients of the OA212 baseline and CFJ airfoils at different AoAs.

of the suction slot and the control effect of the dynamic stall was not positive, but the control effect was the best when the suction slot was placed at $70 \% c$. This is because the more forward the suction slot, the smaller the momentum of the jet injected into the main flow, but the greater the flow velocity around the suction slot; the more backward the suction slot, the greater the momentum of the jet injected into the main flow, but the smaller the flow velocity around the suction slot. Therefore, there is an optimal solution for the suction slot position.

The influence of the suction slot size on the dynamic stall characteristics of the airfoil was studied by varying the size of the suction slot from $0.8 \% c$ to $1.2 \% c$. The pitch motion law of the airfoil and the other parameters of the CFJ airfoil were kept unchanged, and the jet flow coefficient $C_{\mu}=0.08$.
It can be seen from Figure 17 that different suction slot sizes affected the lift coefficient and moment coefficient of the CFJ airfoil; thus, the size of the suction slot has a great influence on the dynamic stall of the rotor airfoil. When the size was too small $(0.8 \% c)$, the overall increase of the lift coefficient was significantly reduced as compared with other sizes; moreover, the area of the lift hysteretic curve was significantly increased, and the range of the linear segment was reduced.

To explore the reasons for this phenomenon, the details of the flow near the suction slot of the CFJ airfoil with different suction slot sizes were further analyzed, as shown in Figure 18. It was found that when the size of the suction slot was small $(0.8 \% c)$, to suck in enough airflow mass to ensure the flow balance, the airflow farther 


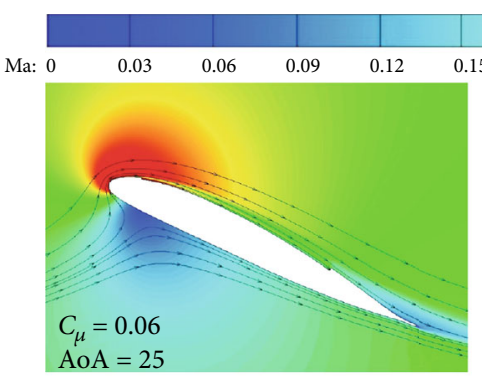

(a) $C \mu=0.06$

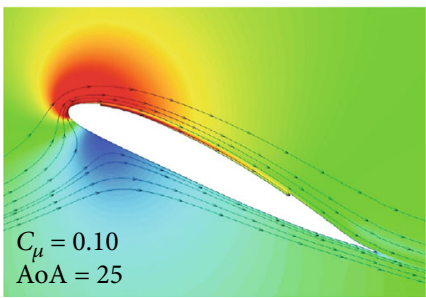

(c) $C_{\mu}=0.10$

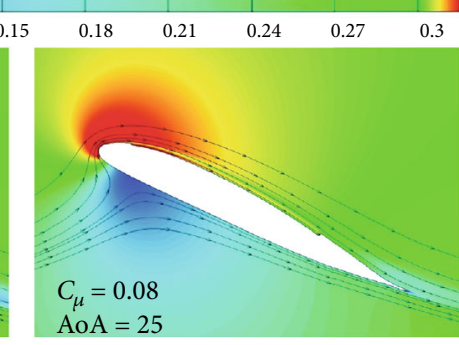

(b) $C_{\mu}=0.08$

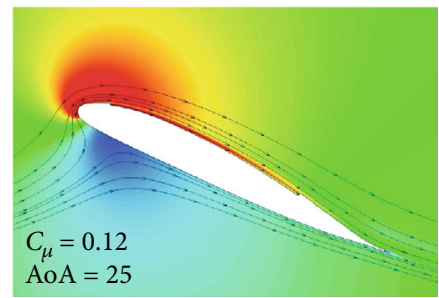

(d) $C \mu=0.12$

FIGURE 11: Mach number contours and streamlines of a OA212 CFJ airfoil with different values of $C_{\mu}$.

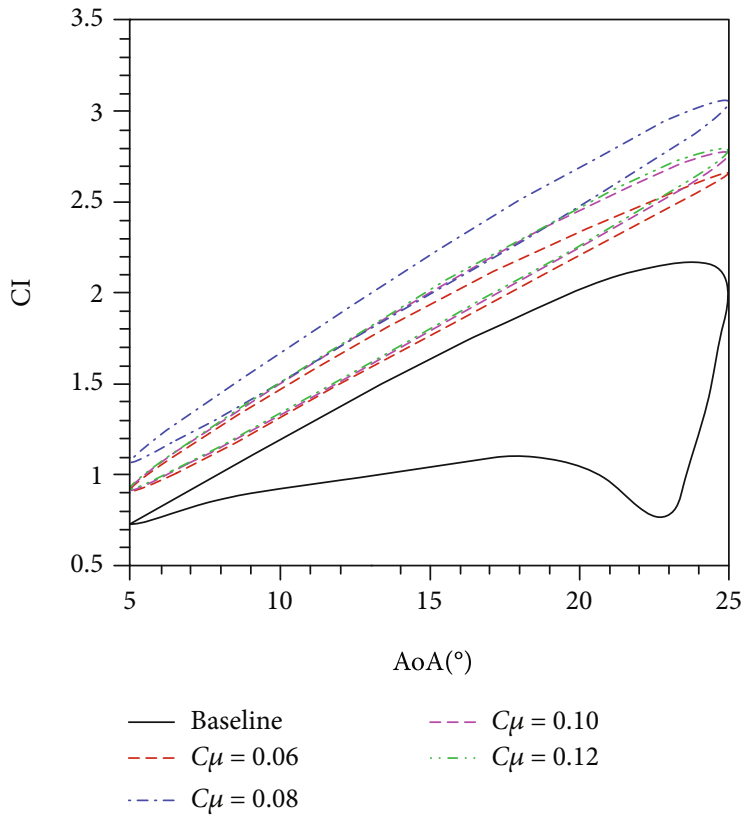

(a) Lift coefficient

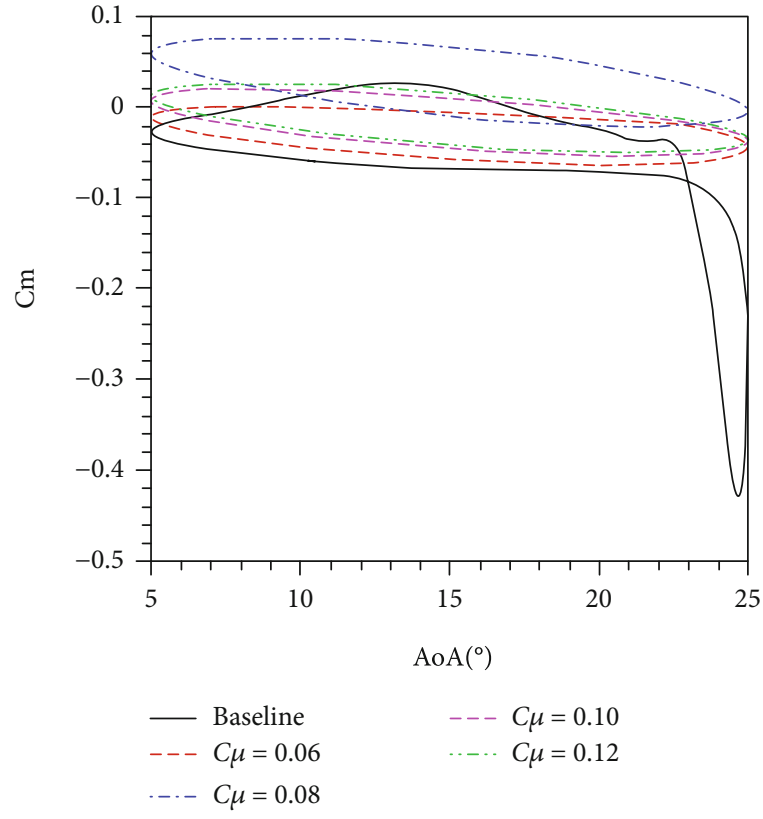

(b) Moment coefficient

FIGURE 12: Lift and moment coefficients of an OA212 CFJ airfoil with different values of $C_{\mu}$.

TABLE 1: The variation of the peak values of the lift and moment coefficients of the airfoil with different values of $C_{\mu}$.

\begin{tabular}{lcccc}
\hline & $C_{l, \max }$ & $\Delta C_{l, \max }$ & $C_{m, \min }$ & $\Delta\left|C_{l, \min }\right|$ \\
\hline Baseline & 2.1728 & - & -0.4256 & - \\
$C_{\mu}=0.06$ & 2.6359 & $21.31 \%$ & -0.0635 & $-85.07 \%$ \\
$C_{\mu}=0.08$ & 2.9934 & $37.77 \%$ & -0.0215 & $-94.95 \%$ \\
$C_{\mu}=0.10$ & 2.7241 & $25.37 \%$ & -0.0536 & $-87.41 \%$ \\
$C_{\mu}=0.12$ & 2.7502 & $26.57 \%$ & -0.0506 & $-88.11 \%$ \\
\hline
\end{tabular}

from the wall was also sucked in. This contracted and blocked the streamline at the suction slot, thereby affecting the control effect of the dynamic stall of the airfoil.

\section{Conclusions}

In this work, the sliding mesh technique was used to solve the unsteady Reynolds-averaged Navier-Stokes equations, and the dynamic stall characteristics of the OA212 rotor airfoil based on CFJ control were numerically simulated. It was verified that the method has the effect of dynamic 


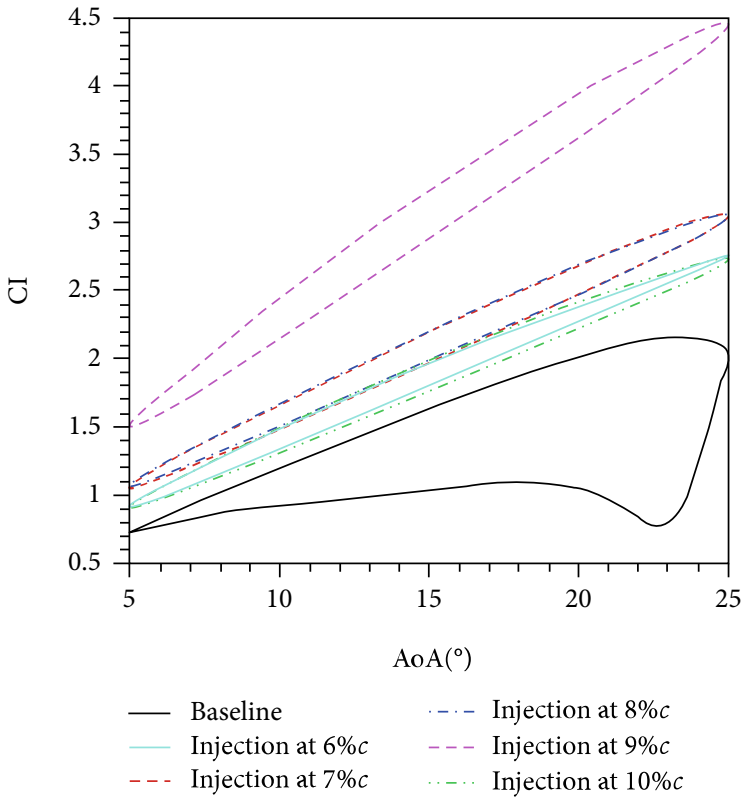

(a) Lift coefficient

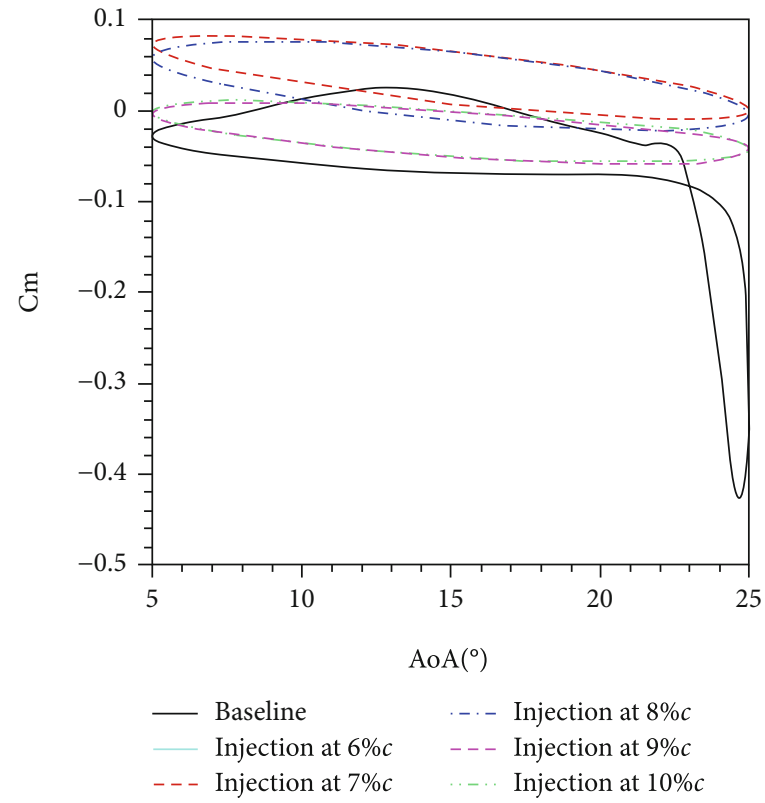

(b) Moment coefficient

Figure 13: Hysteresis curves of the lift and moment coefficients with the AoA at different injection slot positions.

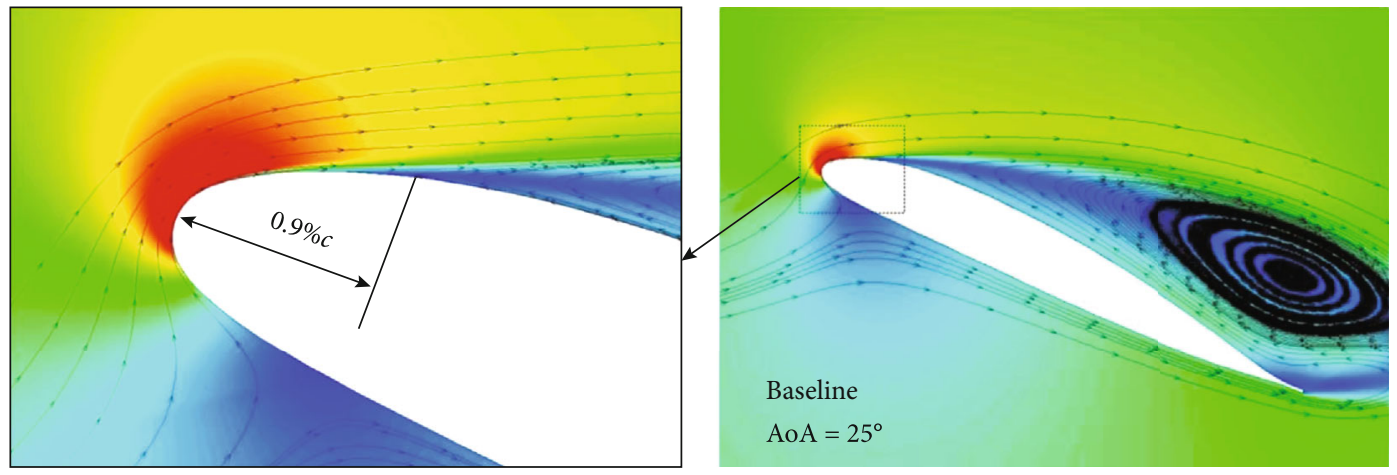

Figure 14: The position of flow separation at the leading edge of the airfoil (baseline, AoA $=25^{\circ}$ ).

stall suppression. On this basis, the influences of the control parameters of the CFJ on the dynamic stall characteristics were studied, and the following conclusions can be drawn.

(1) Numerical simulations of a NACA0012 airfoil dynamic stall case and NACA6415 airfoil flow separation suppression case based on CFJ verified the correctness of the numerical simulation methods for airfoil dynamic stall and CFJ control

(2) Via the numerical simulation of the dynamic stall characteristics of the rotor airfoil based on CFJ control under typical conditions, it was verified that the method can suppress dynamic stall. After applying the CFJ control, the lift coefficient of the airfoil was found to be greatly improved, the negative peak of the moment coefficient was significantly reduced, the area of the hysteresis loop was reduced, and the hysteresis effect was significantly weakened. Moreover, the CFJ was also found to improve the aerodynamic damping characteristics of the airfoil, and the aerodynamic damping represented by the moment coefficient hysteresis curve was positive, thereby increasing the stability of the system

(3) The high-energy airflow injected from the airfoil leading-edge injection slot with CFJ control allowed the energy to transfer from the jet to the main flow in a single direction, thereby enhancing the ability to resist adverse pressure gradients at high AoAs. This suppressed the flow separation, allowing the effect of dynamic stall suppression to be achieved. The stagnation point position of the leading edge of the airfoil was moved backward as compared with that of the baseline airfoil, and the effective AoA was increased. Additionally, the 


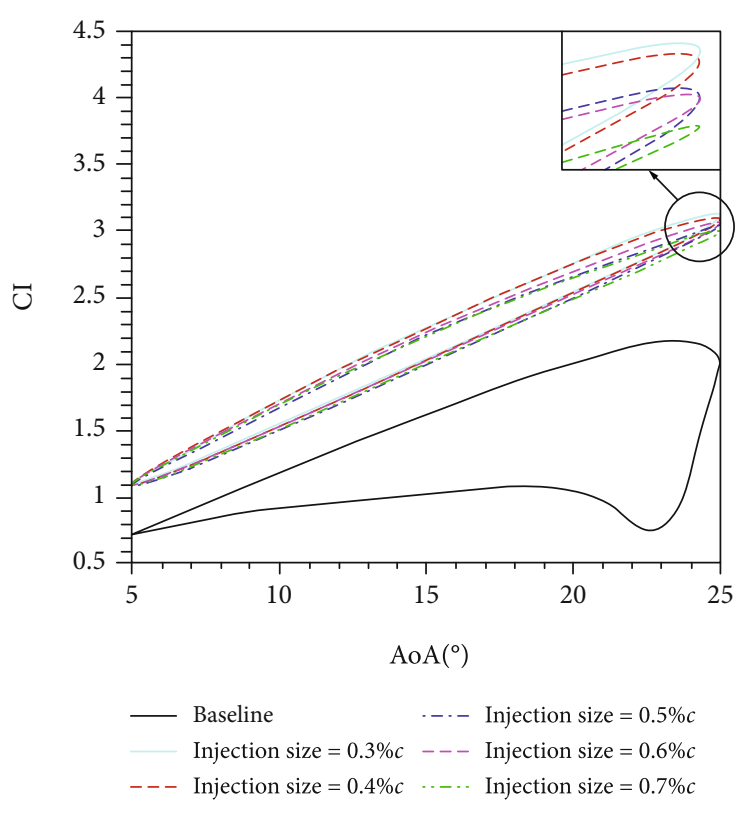

(a) Lift coefficient

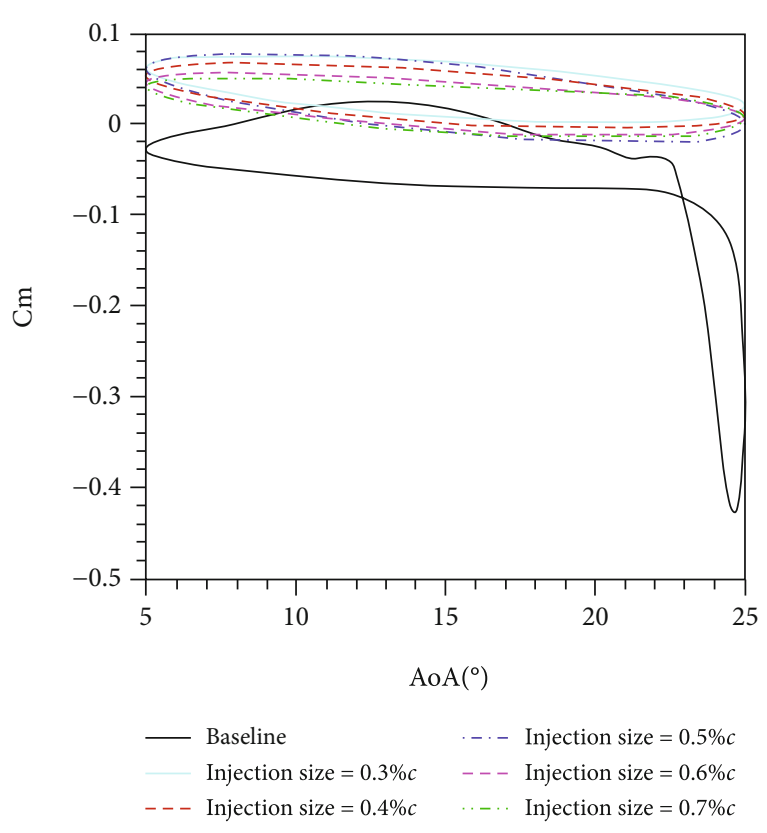

(b) Moment coefficient

Figure 15: Hysteresis curves of lift and moment coefficients with the AoA under different injection slot sizes.

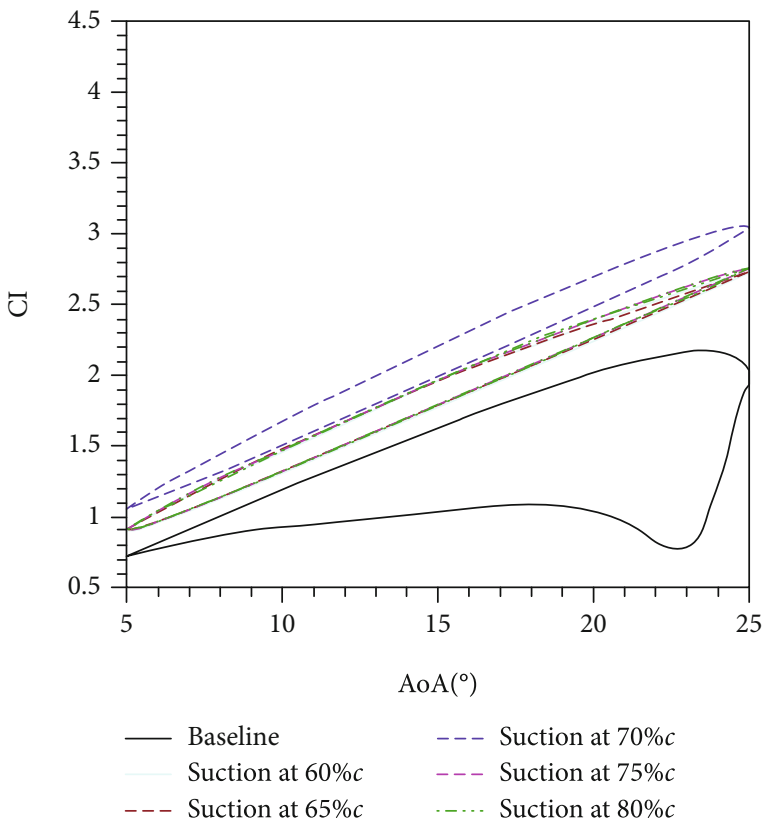

(a) Lift coefficient

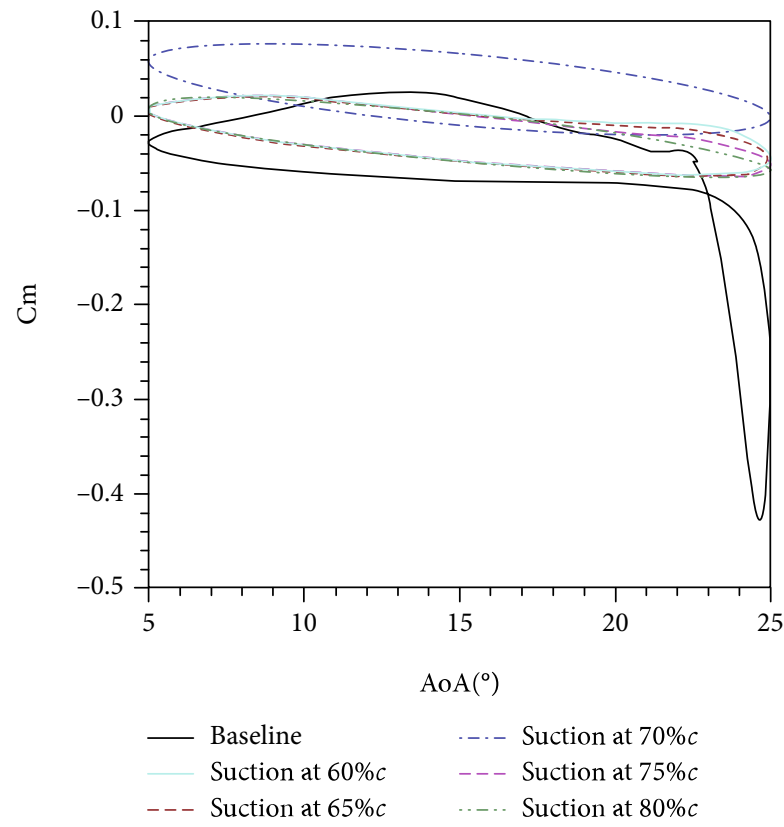

(b) Moment coefficient

Figure 16: Hysteresis curve of the lift and moment coefficients with AoA at different suction slot positions.

airflow velocity of the airfoil suction surface was also faster than that of the baseline airfoil, thereby increasing the lift coefficient

(4) The control effect of the dynamic stall of the rotor airfoil was not found to have a simple positive correlation with the increase of the CFJ momentum coefficient; instead, there was an optimal jet momentum coefficient that optimized the control effect of airfoil dynamic stall. Therefore, on the premise of ensuring the control effect, a smaller jet momentum coefficient should be selected to reduce energy consumption

(5) The position of the CFJ injection slot was found to have a greater influence on the effect of the dynamic stall control of the rotor airfoil. The effect of restraining the flow separation was found to be 


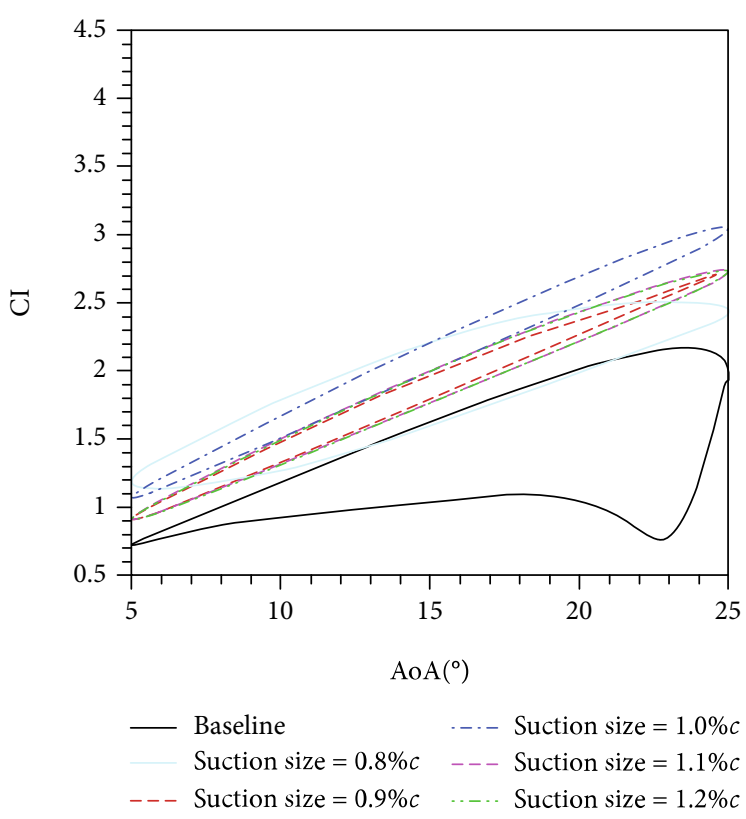

(a) Lift coefficient

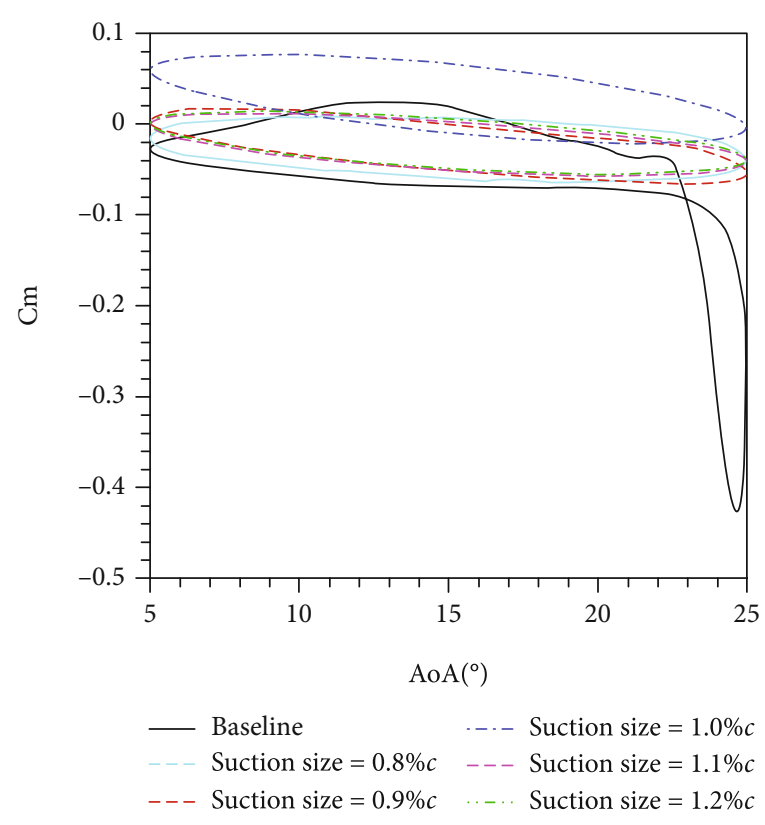

(b) Moment coefficient

FIgURE 17: Hysteresis curves of the lift and moment coefficients with the AoA under different suction slot sizes.

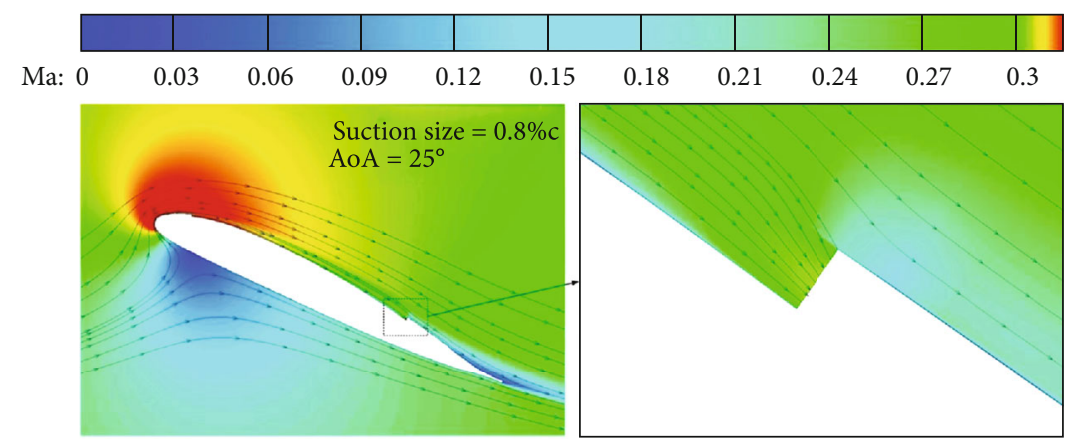

(a) Suction slot size $=0.8 \% \mathrm{c}$

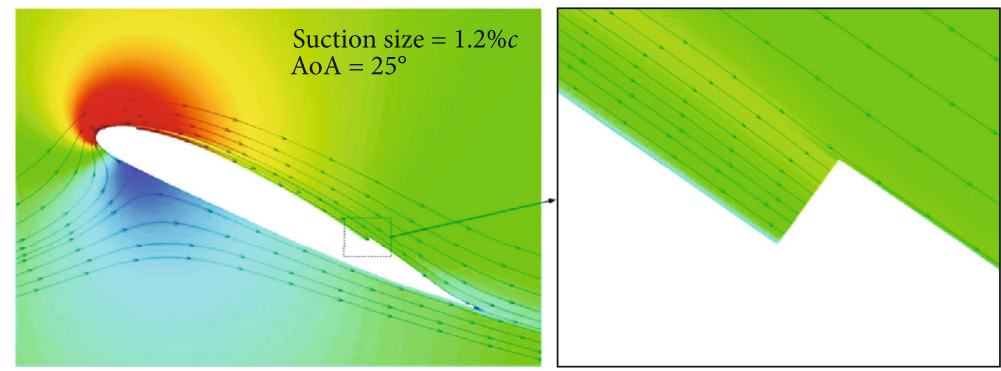

(b) Suction slot size $=1.2 \% \mathrm{c}$

Figure 18: Details of the CFJ airfoil flow field with different suction slot sizes.

the best when the injection slot was arranged at the leading-edge flow separation point and the rotor airfoil was at the maximum AoA. The size of the suction slot was found to have little effect on suppressing the dynamic stall of the airfoil, but it had a slight effect on the lift coefficient of the airfoil at high AoAs. The position of the suction slot was also found to have little effect on suppressing the dynamic stall of the airfoil. The size of the suction slot should not be too small; otherwise, it will be blocked, thereby weakening the effect of dynamic stall suppression

\section{Data Availability}

The data used to support the findings of this study are available from the corresponding author upon request. 


\section{Conflicts of Interest}

The authors declare that there is no conflict of interest regarding the publication of this paper.

\section{Acknowledgments}

The work was supported by the National Natural Science Foundation of China under Grant No. 12072305, the Equipment field pre-research fund under Grant No. 61402060205, the open fund from Rotor Aerodynamics Key Laboratory under Grant No. RAL20190303, the Aeronautics Power Foundation under Grant No. 6141B09050347, and the open fund from State Key Laboratory of Aerodynamics under Grant No. SKLA20180203.

\section{References}

[1] W. Geissler, M. Raffel, G. Dietz, and H. Mai, "Helicopter aerodynamics with emphasis placed on dynamic stall," Wind Energy, pp. 199-204, 2007.

[2] A. T. Conlisk, "Modern helicopter rotor aerodynamics," Progress in Aerospace Sciences, vol. 37, no. 5, pp. 419-476, 2001.

[3] Y. H. Yu, S. Lee, M. A. KW, C. Tung, and C. M. Wang, "Dynamic stall control for advanced rotorcraft application," AIAA Journal, vol. 33, no. 2, pp. 289-295, 1995.

[4] A. H. Lind and A. R. Jones, "Unsteady aerodynamics of reverse flow dynamic stall on an oscillating blade section," Physics of Fluids, vol. 28, no. 7, article 077102, 2016.

[5] T. Schwermer, A. D. Gardner, and M. Raffel, "A novel experiment to understand the dynamic stall phenomenon in rotor axial flight," Journal of the American Helicopter Society, vol. 64, no. 1, pp. 1-11, 2019.

[6] H. R. Karbasian and K. C. Kim, "Numerical investigations on flow structure and behavior of vortices in the dynamic stall of an oscillating pitching hydrofoil," Ocean Engineering, vol. 127, pp. 200-211, 2016.

[7] J. A. Esfahani, E. Barati, and H. R. Karbasian, "Fluid structures of flapping airfoil with elliptical motion trajectory," Computers \& Fluids, vol. 108, pp. 142-155, 2015.

[8] H. R. Karbasian, J. A. Esfahani, and E. Barati, "Effect of acceleration on dynamic stall of airfoil in unsteady operating conditions," Wind Energy, vol. 19, no. 1, pp. 17-33, 2016.

[9] G. J. Leishman, Principles of Helicopter Aerodynamics with CD Extra, Cambridge university press, 2006.

[10] A. Le Pape, M. Costes, F. Richez, G. Joubert, F. David, and J. M. Deluc, "Dynamic stall control using deployable leading-edge vortex generators," AIAA Journal, vol. 50, no. 10, pp. 2135$2145,2012$.

[11] B. Heine, K. Mulleners, G. Joubert, and M. Raffel, "Dynamic stall control by passive disturbance generators," AIAA Journal, vol. 51, no. 9, pp. 2086-2097, 2013.

[12] C. Singh, D. Peake, F. Coton, A. Kokkalis, R. Galbraith, and V. Khodagolian, "The application of air-jet vortex generators to suppress flow separation on helicopter aerofoil sections under quasi-steady and unsteady conditions," 42nd AIAA Aerospace Sciences Meeting and Exhibit, p. 45, 2018.

[13] D. Jeffrey, X. Zhang, and D. W. Hurst, "Aerodynamics of Gurney flaps on a single-element high-lift wing," Journal of Aircraft, vol. 37, no. 2, pp. 295-301, 2000.
[14] M. P. Kinzel, M. D. Maughmer, and E. P. Duque, "Numerical investigation on the aerodynamics of oscillating airfoils with deployable gurney flaps," AIAA Journal, vol. 48, no. 7, pp. 1457-1469, 2010.

[15] D. Han, C. Dong, and G. N. Barakos, "Performance improvement of variable speed rotors by Gurney flaps," Aerospace Science and Technology, vol. 81, pp. 118-127, 2018.

[16] L. W. Carr, M. S. Chandrasekhara, M. C. Wilder, and K. W. Noonan, "Effect of compressibility on suppression of dynamic stall using a slotted airfoil," Journal of Aircraft, vol. 38, no. 2, pp. 296-309, 2001.

[17] R. Balaji, F. Bramkamp, M. Hesse, and J. Ballmann, "Effect of flap and slat riggings on 2-D high-lift aerodynamics," Journal of Aircraft, vol. 43, no. 5, pp. 1259-1271, 2006.

[18] W. Joo, B. S. Lee, K. Yee, and D. H. Lee, "Combining passive control method for dynamic stall control," Journal of Aircraft, vol. 43, no. 4, pp. 1120-1128, 2006.

[19] A. A. Hassan, F. K. Straub, and K. W. Noonan, "Experimen$\mathrm{tal} /$ numerical evaluation of integral trailing edge flaps for helicopter rotor applications," Journal of the American Helicopter Society, vol. 50, no. 1, pp. 3-17, 2005.

[20] R. Jain, H. Yeo, and I. Chopra, "Computational fluid dynamics-computational structural dynamics analysis of active control of helicopter rotor for performance improvement," Journal of the American Helicopter Society, vol. 55, no. 4, p. 42004, 2010.

[21] U. Visconti, W. Eun, J. Kang, J. Lim, J. Sim, and S. J. Shin, "Transient flow analysis and static bench measurements for an active trailing-edge flap," in 24th AIAA/AHS Adaptive Structures Conference, San Diego, California, USA, 2016.

[22] F. K. Straub, V. R. Anand, B. H. Lau, and T. S. Birchette, "Wind tunnel test of the SMART active flap rotor," Journal of the American Helicopter Society, vol. 63, no. 1, pp. 1-6, 2018.

[23] J. Niu, J. Lei, and T. Lu, "Numerical research on the effect of variable droop leading-edge on oscillating NACA 0012 airfoil dynamic stall," Aerospace Science and Technology, vol. 72, pp. 476-485, 2018.

[24] W. Geissler and B. G. van der Wall, "Dynamic stall control on flapping wing airfoils," Aerospace Science and Technology, vol. 62 , pp. 1-10, 2017.

[25] G. Q. Zhao and Q. J. Zhao, "Dynamic stall control optimization of rotor airfoil via variable droop leading-edge," Aerospace Science and Technology, vol. 43, pp. 406-414, 2015.

[26] L. W. Traub, A. Miller, and O. Rediniotis, "Effects of synthetic jet actuation on a ramping NACA 0015 airfoil," Journal of Aircraft, vol. 41, no. 5, pp. 1153-1162, 2004.

[27] Q. J. Zhao, Y. Y. Ma, and G. Q. Zhao, "Parametric analyses on dynamic stall control of rotor airfoil via synthetic jet," Chinese Journal of Aeronautics, vol. 30, no. 6, pp. 1818-1834, 2017.

[28] G. Zhao, Q. Zhao, Y. Gu, and X. Chen, "Experimental investigations for parametric effects of dual synthetic jets on delaying stall of a thick airfoil," Chinese Journal of Aeronautics, vol. 29, no. 2, pp. 346-357, 2016.

[29] M. L. Post and T. C. Corke, "Separation control using plasma actuators: dynamic stall vortex control on oscillating airfoil," AIAA Journal, vol. 44, no. 12, pp. 3125-3135, 2006.

[30] T. C. Corke, P. O. Bowles, C. He, and E. H. Matlis, "Sensing and control of flow separation using plasma actuators," Philosophical Transactions of the Royal Society a-Mathematical Physical and Engineering Sciences, vol. 369, no. 1940, pp. 1459-1475, 2011. 
[31] A. J. Lombardi, P. O. Bowles, and T. C. Corke, "Closed-loop dynamic stall control using a plasma actuator," AIAA Journal, vol. 51, no. 5, pp. 1130-1141, 2013.

[32] H. S. Yang, G. Y. Zhao, H. Liang, and B. Wang, "Research progress on influence factors of airfoil dynamic stall and flow control," Acta Aeronauticaet Astronautica Sinica, vol. 41, no. 8, pp. 23605-023605, 2020.

[33] G. C. Zha and C. Paxton, "A novel airfoil circulation augment flow control method using co-flow jet," in 2nd AIAA Flow Control Conference, Portland, Oregon, USA, 2004.

[34] G. C. Zha, B. F. Carroll, C. D. Paxton, C. A. Conley, and A. Wells, "High-performance airfoil using coflow jet flow control," AIAA Journal, vol. 45, no. 8, pp. 2087-2090, 2007.

[35] G. C. Zha and W. Gao, "Analysis of jet effects on co-flow jet airfoil performance with integrated propulsion system," 44th AIAA Aerospace Sciences Meeting and Exhibit, 2006.

[36] B. Dano, G. C. Zha, and M. Castillo, "Experimental study of co-flow jet airfoil performance enhancement using discreet jets," 49th AIAA Aerospace Sciences Meeting Including the New Horizons Forum and Aerospace Exposition, 2011.

[37] B. Wang, B. Haddoukessouni, J. Levy, and G. C. Zha, "Numerical investigations of injection-slot-size effect on the performance of coflow jet airfoils," Journal of Aircraft, vol. 45, no. 6, pp. 2084-2091, 2008.

[38] A. Lefebvre and G. C. Zha, "Numerical simulation of pitching airfoil performance enhancement using co-flow jet flow control," in 31st AIAA Applied Aerodynamics Conference, p. 2517, San Diego, CA, USA, 2013.

[39] H. Y. Xu, C. L. Qiao, and Z. Y. Ye, "Dynamic stall control on the wind turbine airfoil via a co-flow jet," Energies, vol. 9, no. 6 , p. 429, 2016.

[40] H. Q. Yang, H. Y. Xu, and Z. Y. Ye, "Study on the flow control of the airfoil dynamic stall using the co-flow jet," Advance in Aeronautical Science and Engineering, vol. 9, no. 4, pp. 566576, 2018.

[41] X. D. Yang, W. R. Jiang, and S. L. Zhang, "Analysis of co-flow jet effect on dynamic stall characteristics applying to rotor airfoils," IOP Conference Series: Materials Science and Engineering, vol. 491, 2019, Bangkok, Thailand, 2019no. 1, Article ID 012010, 2019.

[42] J. Blazek, Computational Fluid Dynamics: Principles and Applications, Butterworth-Heinemann, 2015.

[43] A. Jameson, "Time dependent calculations using multigrid, with applications to unsteady flows past airfoils and wings," in 10th Computational Fluid Dynamics Conference, 1991Honolulu, HI, USA.

[44] P. R. Spalart and S. R. Allmaras, "A one-equation turbulence model for aerodynamic flows," 30th aerospace sciences meeting and exhibit, p. 439, 1992.

[45] M. A. KW, S. L. Pucci, M. C. WJ, and L. W. Carr, An Experimental Study of Dynamic Stall on Advanced Airfoil Section. Volume 2: Pressure and Force Data, 1982.

[46] B. P. E. Dano, D. Kirk, and G. C. Zha, "Experimental investigation of jet mixing of a co-flow jet airfoil," in Flow Control Conference, vol. 28, Chicago, Illinois, 2010.

[47] A. Lefebvre, B. Dano, W. B. Bartow, M. D. Fronzo, and G. Zha, "Performance and energy expenditure of coflow jet airfoil with variation of mach number," Journal of Aircraft, vol. 53, no. 6, pp. 1757-1767, 2016.
[48] Z. H. Han, W. P. Song, and Z. D. Qiao, "Numerical simulation of active dynamic stall control on an OA212 rotor airfoil," Acta Aerodynamica Sinica, vol. 27, no. 6, pp. 639-644, 2007.

[49] T. C. Corke and F. O. Thomas, "Dynamic stall in pitching airfoils: aerodynamic damping and compressibility effects," Annual Review of Fluid Mechanics, vol. 47, pp. 479-505, 2015. 